\title{
EDITORIAL
}

\section{Adapting to circumstances}

\author{
The traditional 'frequentist' approach to clinical trials has been the gold standard for several \\ decades, but its rigour has a cost: lack of flexibility. With regulatory authorities increasingly \\ open to alternatives, careful adoption of adaptive trial designs could provide some much- \\ needed improvement in the circumstances of innovative drug development.
}

Sixty years ago, the first truly randomized controlled clinical trials were started. The 1948 publication of one of these trials - demonstrating that streptomycin therapy and bed rest was more effective than bed rest alone in treating patients with pulmonary tuberculosis - represented a landmark in clinical research. Today, clinical trials must generally have several features for the results they produce to be accepted as reliable and valid, including appropriate controls and randomization, predefinition of the patient population and outcomes to be evaluated, and blinding. Indeed, the data accumulating during a trial is generally kept secret from those involved until its completion, with the exception of independent data and safety monitoring boards.

However, although such features are crucial for ensuring the integrity of clinical trials based on traditional 'frequentist' statistics, there is a major downside: inflexibility. As a result, trials tend to be overly large, and some patients are unnecessarily exposed to inferior experimental therapies. Furthermore, on learning the results of the trial, it can be apparent that more could have been learned if the patients had been assigned in a different manner; for example, in a dose-finding study where the dose-response curve differs considerably from what was anticipated ${ }^{1}$.

Awareness of these limitations of traditional clinical trial designs, and their adverse impact on the already sky-high costs of clinical development, has led to growing interest in innovative alternatives, such as those based on Bayesian statistical designs ${ }^{1}$. A key feature of such approaches is that they allow adaption of the trial design in the light of information accrued during the trial. So, whereas in a traditional clinical trial, patients might be allocated between one of two different therapies, and a decision made at the end of the trial as to which is more effective, in an adaptive clinical trial, by contrast, data can be used as they become available to modify the trial design in various ways. For example, randomization can be imbalanced to favour better-performing therapies, or the trial population can be focused on patient subsets that are responding better to treatment. Dose-finding studies based on adaptive designs can also readily assess considerably more dose levels than traditional designs, thereby aiding the identification of the optimal dose for large-scale studies. Overall, such approaches have the potential to offer significant efficiency, cost and ethical advantages over traditional approaches, while still allowing statistically meaningful conclusions to be drawn.

Given such potential advantages, why are adaptive clinical trials not already commonplace? Well, one simple reason is that they're challenging. In order to provide meaningful results, adaptive trials can be considerably more complex to design, implement and analyse than traditional trials. However, major advances in computational power and techniques have already gone a long way towards addressing this challenge. Arguably, the key issue now is the attitude of regulatory authorities to adaptive trials, or perhaps even more importantly the pharma industry's understanding of the attitude of these authorities.

So the recent announcement from the US FDA ${ }^{2}$ that it will develop guidelines to facilitate the use of adaptive clinical trials could be a powerful catalyst for their more widespread application. Nevertheless, even with such regulatory encouragement, selling the concept of adaptive designs to a sceptical public could be a considerable challenge, especially for late-stage clinical trials intended to demonstrate drug safety and efficacy.

It is therefore crucial at this stage to bear in mind that although adaptive trials have many advantages, their design and logistics need careful and expert consideration in order to preserve high scientific standards. Given this, perhaps it might be wise if new applications of adaptive trial designs focused initially on the earlier phases of drug development, such as dose-finding studies, while both regulators and developers gain more experience of the associated challenges and issues. There is no doubt that innovative adaptive trial designs represent a much-needed opportunity to improve the effectiveness of drug development, but enthusiasm for such designs must be coupled with a realistic assessment of the present ability to implement them with integrity.

\footnotetext{
. Berry, D. A. Nature Rev. Drug Discov. 5, 27-36 (2006)

. Gottlieb, S. [online], < http://www.fda.gov/oc/speeches/2006 trialdesign0710.html > (2006).
} 